\title{
Detection of Pre-stage of Epileptic Seizure by Exploiting Temporal Correlation of EMD Decomposed EEG Signals
}

\author{
Mohammad Zavid Parvez, Manoranjan Paul, and Michael Antolovich \\ School of Computing and Mathematics \\ Charles Sturt University, Australia \\ Email: \{mparvez,mpaul,mantolovich\}@csu.edu.au
}

\begin{abstract}
Epilepsy is one of the common neurological disorders characterized by a sudden and recurrent malfunction of the brain that is termed "seizure", affecting over 50 million individuals worldwide. The Electroencephalogram (EEG) is the most influential technique in detection of epileptic seizures. In recent years, many research works have been devoted to the detection of epileptic seizures based on analysis of EEG signals. Despite remarkable work on seizure detection, there is no generic seizure detection scheme which performs reasonably well for different patients and different brain locations. In this paper we present a generic approach for feature extraction of preictal (pre-stage of seizure onset) and interictal (period between seizures) EEG signals using empirical mode decomposition (EMD) along with discrete cosine transformation (DCT) by exploit temporal correlation for detection of preictal phase of epileptic seizure. Then least square support vector machine is applied on the features for classifications. Results demonstrate that our proposed method outperforms the state-of-the-art methods in terms of sensitivity, specificity and accuracy to classify preictal and interictal EEG signals to the benchmark dataset extracted from different brain locations of different patients.
\end{abstract}

Keywords-EEG, Epilepsy, Seizure, EMD, DCT, LS-SVM.

\section{INTRODUCTION}

Seizure is simply the medical condition or neurological disorder in which too many neurons are excited in the same time caused by brain injury or by an imbalance of chemical in the brain that is characterized predominantly by unpredictable interruptions of normal brain function. Epilepsy is another medical condition characterized by spontaneously recurrent seizures [1]. Epilepsy may lead to many injuries such as fractures, submersion, burns, motor vehicle accidents and even death. Approximate $1 \%$ of the total population develops epilepsy [2]. It is possible to prevent epileptic seizure with high sensitivity (i.e., detecting the preictal signal) if electrical changes in the brain that occur prior to the onset of an actual seizure can be detected.

The human brain processes sensory information received by external/internal stimuli. In the brain, neurons exploit chemical reaction to generate electricity to control different bodily actions and this ongoing electrical activity can be recorded graphically with an Electroencephalogram (EEG). It is usually accepted that EEG recordings are very reliable in the diagnosis of epilepsy. EEG signals represent the non-linear nature of the recorded signals in which there are two key terms, namely, 'state' and 'dynamics'. The state defines the signal at a given time and dynamics is characterized by the changing rate of the signal over time [3]. EEG signals from an epileptic patient can be divided into five periods or stages (i) non-seizure period- no epileptic syndrome is visible, (ii) ictal period-actual seizure period, normally duration is 1 to 3 minutes (iii) preictal period- 30 minutes to 60 minutes before ictal period, (iv) post-ictal period- 30 minutes after ictal period, and (v) interictal period- period between post-ictal period to pre-ictal period. Some portion of the interictal period, which does not have any epileptic syndrome, can be defined as a non-seizure period. The most common way to assume seizure onset before it becomes clinically apparent is to analyse the preictal state of the EEG signals. It is emphasized that determining the preictal signal over time is highly significant for gaining accurate seizure prediction results as the preictal signal is considered as the transition point between the interictal and ictal period. With improving technologies and the increase in the number of quality channels, it is important to realize patterns that are potentially involved EEG signals across a range of temporal scales [4].

Existing feature extraction and classification methods based on linear univariate techniques [3], eigenspectra of space delay correlation and covariance matrices [4], Hilbert-Huang transform [5], and autoregressive modeling with least-squares parameter estimator [6] were employed for the detection of preictal and interictal in EEG signals. Rasekhi et al. [3] computed multiple linear univariate features in one feature space and classified the feature space using machine learning methods and predicted epileptic seizures by classifying preictal or non-preictal states. Williamsona et al. [4] derived the eigenspectra of space-delay correlation and covariance matrices from 15-s blocks of EEG data at multiple delay scales and used support vector machine (SVM) to classify preictal and interictal signals. Duman et al. [5] decomposed EEG signals into intrinsic mode functions (IMFs) and the first 5 IMFs were used to obtain features for classification of preictal and interictal EEG signals. Chisci et al. [6] proposed solution relied on a novel autoregressive modelling of the EEG signals and combined a leastsquares parameter estimator for EEG feature extraction along with a SVM for binary classification between preictal and interictal states. According to the classification results of preictal and interictal EEG signals 
with respect to sensitivity, Chisci et al. [6] proposed technique carried good sensitivity compared to the above mentioned techniques [4][5] using dataset [7]. However, Rasekhi et al. [3] obtained 79.3\% sensitivity for their own dataset.

Other feature extraction and classification methods based on wavelet [8]-[10] and Fourier transformation [11] have been proposed for the detection of seizure and nonseizure EEG signals. Panda et al. [8] computed various features like energy, entropy, and standard deviation (STD) using discrete wavelet transformation (DWT) and used SVM as a classifier. Dastidar et al. [9] applied wavelet transformation to decompose the EEG signals into different ranges of frequencies and then extracted three features, such as STD, correlation dimension, and the largest Lyapunov exponent (quantifying the non-linear chaotic dynamics of the signals) and applied different methods for classification. Ocak [10] proposed a fourth level wavelet packet decomposition method to decompose the normal and epileptic EEG epochs to various frequency-bands and then used genetic algorithms to find optimal feature subsets which maximize the classification performance. Polat et al. [11] extracted features using fast Fourier transform (FFT) and then classified using a decision making classifier.

Many techniques have been proposed for correct seizure detection. Among the existing techniques, Bajaj et al. 's [12] technique is the newest and pre-eminent in terms of performance and it can also measure non-linear dynamics of the EEG signals properly. They extracted amplitude modulation (AM) and frequency modulation (FM) bandwidths as features using IMFs which are generated from empirical mode decomposition (EMD) technique for small dataset [15] which consists of five subsets, each containing 100 single-channel EEG signals with 23.6 seconds. They obtained 98.0 to $99.5 \%$ and 99.5 to $100 \%$ accuracy through least squares-SVM (LS-SVM) [13] using radial basis function (RBF) kernel and Morlet kernel, respectively.

Bajaj et al. [12] have decomposed EEG signals into different IMFs and these IMFs are then used for feature extraction. The features exhibited almost perfect classification accuracy for the small dataset [15] of seizure and non-seizure EEG signals. However, their performance was below the expected result using large dataset [7] for preictal and interictal EEG signal classification because the distributions of AM and FM were significantly overlapped in large dataset [7]. As the IMF represents distinguishing characteristics of preictal and interictal signal separation, our proposed method uses IMFs for different feature extraction by exploiting temporal correlation.

In the experiment, a large dataset [7] for comparing preictal and interictal EEG signals for seizure prediction from two different locations, namely, Frontal and Temporal lobe of the human brain (see sample signals of Frontal lobe in Fig. 1) were used. Fig. 1 demonstrate the non-abrupt phenomena (i.e., not easily distinguishable between preictal and interictal based on amplitude and frequency) of the preictal and interictal signal for the Frontal lobe in a large dataset [7]. Before feature extraction, a pre-processing method on raw EEG signals may play a key role in improving performance of the method as EEG signals sometimes contain line noise and other artifacts due to muscle and body movements. Notch filter and independent component analysis (ICA) method are recommended to remove the line noise and artifacts respectively [16]-[18].

In this paper, we propose a method based on ICA for removing artifact, then exploit temporal correlation of the signal by applying EMD decompose and discrete cosine transformation (DCT) techniques to extract features. These features are used as an input to LS-SVM for classification of preictal and interictal EEG signals. The experimental results show that extracted features provide better classification accuracy compared to the existing state-of-the-art method [12] for classification of preictal and interictal EEG signals using large sized benchmark dataset [7] in different brain locations.

The paper is organized as follows: the data formation is described in section 2 , the detailed proposed method with feature extractions, and classifications techniques are described in section 3; the detailed experimental results are explained in section 4 , while section 5 concludes the paper.

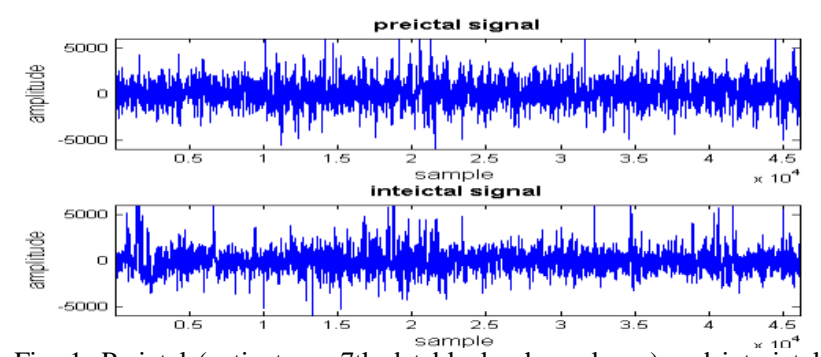

Fig. 1: Preictal (patient one,7th datablock, channel one) and intericta (patient one, 95th datablock, channel one) of Frontal lobe signals from lager dataset [7].

\section{DATASET FORMATION}

The dataset used in the paper was recorded at the Epilepsy Centre of the University Hospital of Freiburg, Germany [7]. The database contains invasive EEG recordings of 21 patients suffering from medically intractable focal epilepsy. The data was obtained by the Neurofile NT digital video EEG system with 128 channels, $256 \mathrm{~Hz}$ sampling rate, and 16 bit analogue-todigital converter. According to dataset, the epileptic EEG signals can be classified into ictal, preictal, and interictal. Normally, duration of ictal varies from a few seconds to 2 minutes. The ictal records contain epileptic seizures with at least 50 minutes of preictal signal preceding each seizure. The median time period between the last seizure and the interictal signal is 5 hours and 18 minutes, and the median time period between the interictal signal and the first following seizure is 9 hours and 36 minutes [4]. Suggested by [7], preictal has been treated as the 3 minutes prior to seizure onset. Three minutes from each interictal signal was also taken from the dataset to provide a comparable sized sample. Three minutes are taken from the 7th minute of an interictal signal. In this experiment, EEG signals from 12 patients where 6 patients for Frontal lobe signals and 6-patients for Temporal lobe signals with focal electrodes were used. 

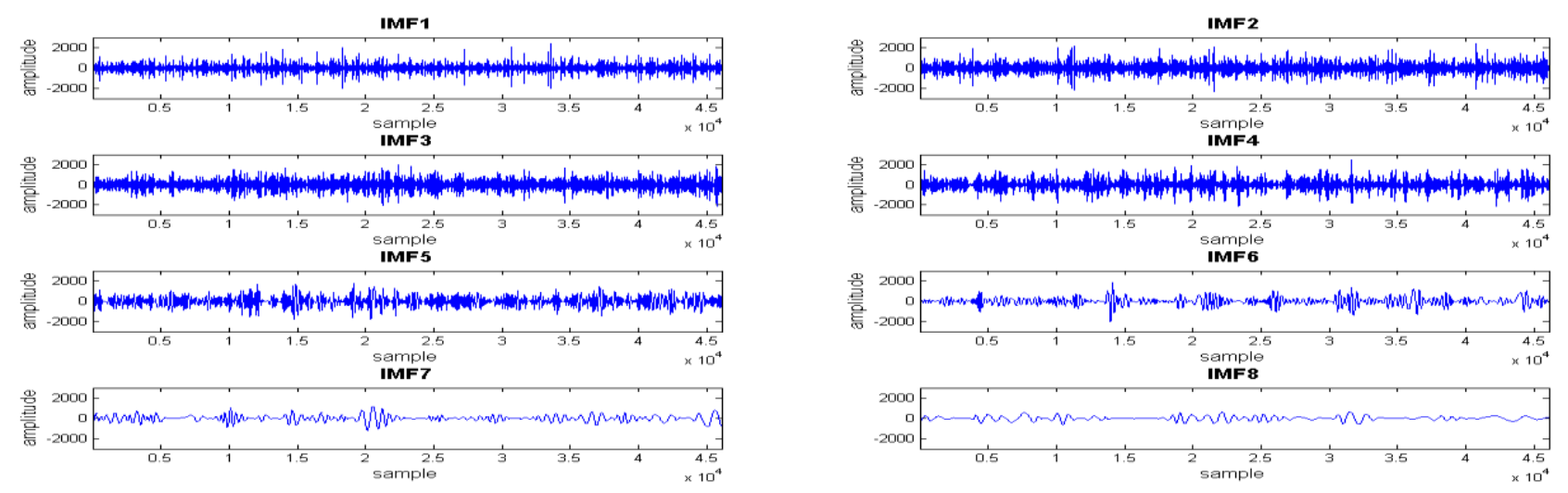

Fig. 2: Extracted IMFs by EMD from preictal signal from Frontal lobe (patient one, 7th datablock, channel one).

\section{PROPOSED METHOD}

A number of researchers [12] indicate that the distinguishing features of ictal (or preictal) are in the high frequency components compared to interictal EEG signals. Thus, theoretically, the features extracted from the first few IMFs should be well enough to classify ictal or preictal EEG signals from interictal signals. For further exploiting high frequency components of the signals for feature extraction, we take a subset of high frequency coefficients after applying DCT on the IMF. The proposed seizure detection technique consists of pre-processing, feature extraction, and classification. A feature extraction method based on well established decomposition such as EMD is proposed. IMFs are generated using EMD and then DCT is applied to the temporally correlated IMFs signals for pre-stage of epileptic seizure detection. A LSSVM classifier was used for the large dataset [7] of 12 patients captured from the Frontal and Temporal lobes for comparison against the state-of-the-art method [12].

\section{A. Preprocessing}

The intention of data pre-processing is to improve the levels of signals of interest, while attenuating or rejecting unwanted signals in the recordings that are marked by artifacts. Thus, the ICA method was applied to these signals to remove artifacts. ICA has emerged as a novel and promising new tool for performing artifact corrections on EEG signals [16]-[18]. Muscle and body movement artifacts were significantly reduced.

\section{B. Features Extraction}

The main strength of EMD is its ability to analyse nonlinear and non-stationary signals like EEG signals. By applying EMD on a signal, we can decompose the signal into a finite number IMFs, which are ordered from higher frequency components to lower frequency components (see in Fig. 2). The number of IMFs in a signal depends on the local characteristics of the signal rather than a predefined number of IMFs. Thus, IMFs can be used in classification applications where the original signals are non-linear and non-stationary and distinguishing features exist in the different frequencies/amplitudes. Each IMF satisfies two the following conditions: (i) the number of extrema and the number of zero crossings are identical or differ at most by one and (ii) the mean value between the upper and the lower envelope is equal to zero at any time.

The EMD algorithm can be summarized as follows:

1. Extract the extrema (minima and maxima)of the signal $x(t)$.

2. Interpolate between minima and maxima to obtain $\ell_{\min }(t)$ and $\ell_{\max }(t)$.

3. Calculate local mean $m(t)=\left[\ell_{\text {min }}(t)+\ell_{\text {max }}(t)\right] / 2$.

4. Extract the detail $d(t)=x(t)-m(t)$.

5. Check that $d(t)$ is an IMF according to the conditions which are mentioned above. If yes, repeat the procedure from step 1 on the residual signal $r(t)=x(t)-m(t)$. If no, replace $x(t)$ with $d(t)$ and repeat the procedure from step 1.

The pictorial scenario of generating IMFs is shown in Fig. 2. The signal $x(t)$ can be represented as a combination of IMFs and residual component:

$$
x(t)=\sum_{i=1}^{L} d_{i}(t)+r_{L}(t)
$$

where, $d_{i}$ and $r_{L}$ are the $i$-th IMF and $L$-th residual signal.

We have observed that features extracted from the first three IMFs provide the best classification results with no significant reduction in classification accuracy. Therefore, in our experimental results we have only investigated the first three IMF from the EMD. Note that when we use $i$-th number of IMF for feature extraction, we do not need to extract subsequent IMFs (i.e., $i+1$ th, $i+2$ th, etc.) to overall reduce computational time.

The features extraction process using EMD decomposed IMF and DCT is summarized as follows:

i. Take three-minute EEG signal from each channel and apply ICA for artifacts remove.

ii. Apply EMD on artifact free EEG signal and then consider each IMF and divide into 60 second blocks. 


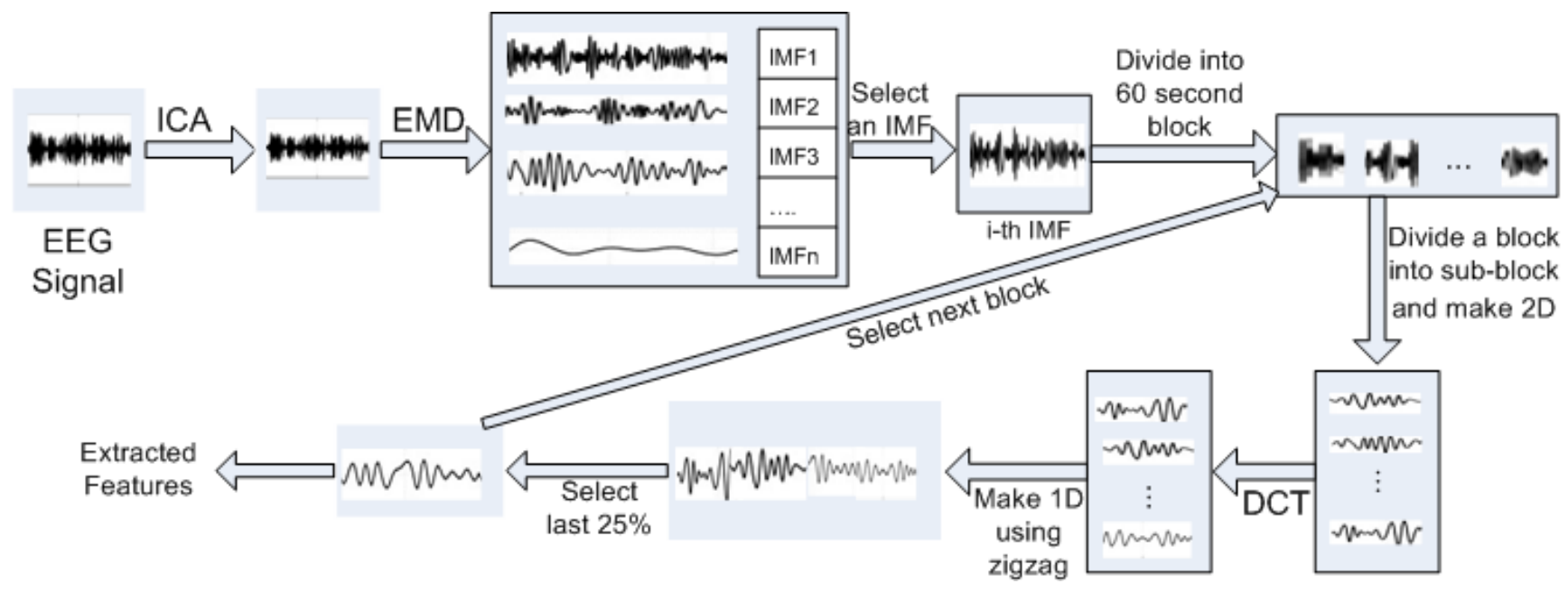

Fig. 4: Features extraction procedure to exploit temporal correlation through ICA, EMD and DCT.

iii. Divide each block again into 2 second sub-blocks to form a matrix for exploiting temporal correlation.

iv. Apply DCT on each matrix and form a 1D using zigzag manner.

v. Take $25 \%$ of high frequency DCT coefficients and calculate energy and entropy.

vi. Repeat the procedure from (iii) to (v) until end of available sub-blocks of a signal.

vii. Calculate average value from $\mathrm{k}$ number of maximum energy and entropy separately (in our experiment we use $\mathrm{k}=2$ ).

viii. Repeat the procedure from (i) to (vii) until end of available signals.
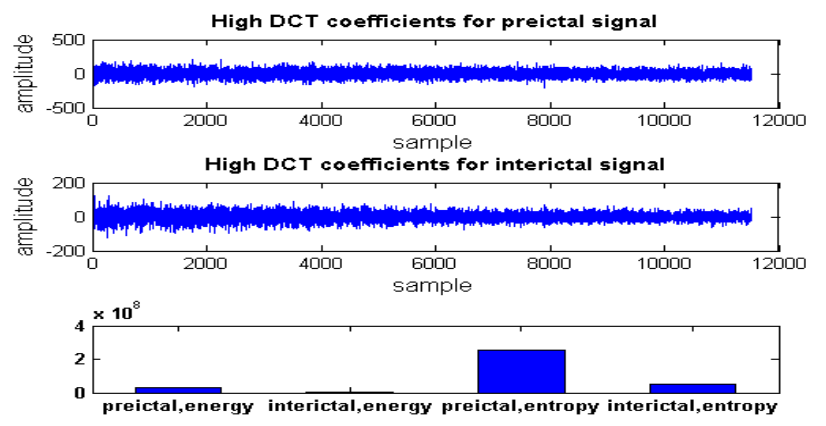

Fig. 3: The first IMFs of ictal and interictal signals with the extracted features; the top two rows represent the first IMF from the preictal and interictal signals of Frontal lobe; bottom row represents Energy of the first IMF and Entropy of the same IMF of preictal and interical EEG signals.

Energy and entropy are determined using $25 \%$ of high DCT co-efficients for each block since high DCT coefficients carry distinguishable features to classify preictal and interictal EEG signals. Note that energy gives us the signal strength and entropy provides uncertainty of the signals. For preictal, the values of energy and entropy are normally higher compared to that of interictal signal as shown in Fig. 3. Thus, an average of up to the 2nd maximum energy and entropy from preictal and interictal signals are considered. The average values of energy and entropy are used as input for the LS-SVM classifier for preictal and interictal classification.

\section{Classifier}

Two features have been extracted, namely energy and entropy, from transformation/decomposition techniques. To classify preictal and interictal signals, a classifier was required. The goal of a classifier is to find patients states such as preictal (class 1) and interictal (class 2) using machine learning approaches with cross-validation. The challenge is to find the mapping that generalizes from training sets to unseen test sets. For the cross-validation, data were partitioned into training and test sets. This experiment a 10-fold cross-validation was used.

Various features from EMD and DCT were extracted to classify the preictal and interictal signals. An SVMbased classifier was used as it is one of the best classifiers for EEG signal analysis [13]. SVM is a potential methodology for solving problems in linear and nonlinear classifications, function estimation, and kernel based learning methods [19]. It can minimize the operational error and maximize the margin hyperplane, as a result it will maximize the classification performance [19]. A major drawback of SVM is its higher computational burden of the constrained optimization programming, however, LS-SVM can solve this problem [21]. LS-SVM [14] is an extended version of SVM and it is closely related to regularization networks and Gaussian processes, and it also has primal-dual interpretations [20].

The classifier is a LS-SVM, which learns nonlinear mappings from the training set features $\{x\}_{i}=1 \ldots n_{T}$, where $n_{T}$ is the number of training features in the patient's state, preictal $(+1)$ and interictal $(-1)$. Let $\left\{y_{i}\right\}_{i=C_{1,2}}$ designate the LS-SVM validation test outputs mapping to class 1 or class 2. The equation of LS-SVM can be defined in [12] as:

$$
f(x)=\operatorname{sign}\left[\sum_{i=1}^{N} \alpha_{i} y_{i} K\left(x, x_{i}\right)+c\right]
$$

where $K\left(x, x_{i}\right)$ is a kernel function, $\alpha_{i}$ are the Lagrange multipliers, $c$ is the bias term, $x_{i}$ is the training input, and 
$y_{i}$ is the training output pairs. RBF is used in these experiments and RBF can be defined in [12] as:

$$
k\left(x, x_{i}\right)=\exp \left(-\left\|x-x_{i}\right\|^{2} / 2 \sigma^{2}\right)
$$

where $\sigma$ controls the width of the RBF function.

\section{EXPERIMENTAL RESULTS}

Our target is to classify preictal and interictal EEG signals using LS-SVM with RBF kernel, where the values of regularization and kernel parameters are generated during cross-validation. In this paper, EMD and DCT based methods have been proposed based on the new features of preictal and interictal EEG signals captured from Frontal and Temporal lobes. After testing all features, sensitivity, specificity, and accuracy [12] were calculated. The sensitivity, specificity, and accuracy are defined as:

$$
\begin{aligned}
& \text { Sensitivity }=\frac{T P}{T P+F N} \times 100 \\
& \text { Specificity }=\frac{T N}{T N+F P} \times 100 \\
& \text { Accuracy }=\frac{T P+T N}{T P+T N+F P+F N} \times 100
\end{aligned}
$$

where $T P$ and $T N$ represents the total number of detected true positive events and true negative events respectively. The $F P$ and $F N$ represent false positive and false negative respectively.

TABLE 1: SENSITIVITY, SPECIFICITY, AND ACCURACY FOR DIFFERENT FEATURES OF PREICTAL AND INTERICTAL EEG SIGNALS FROM FRONTAL AND TEMPORAL LOBE USING DATASET [7] FOR RBF KERNEL.

\begin{tabular}{|l|l|l|l|l|l|l|}
\hline \multirow{3}{*}{ Lobe } & & $\begin{array}{l}\text { Techniqu } \\
\boldsymbol{e} \\
{[\mathbf{1 2 ]}]}\end{array}$ & $\begin{array}{l}\text { Techni } \\
\text { que } \\
{[\mathbf{2 2}]}\end{array}$ & \multicolumn{2}{|l|}{ Proposed } \\
\cline { 3 - 7 } & & $A M-F M$ & $D W T$ & $I M F I$ & $I M F 2$ & $I M F 3$ \\
\hline \multirow{3}{*}{ Frontal } & SEN & 27.6 & $\underline{97.1}$ & 100.0 & 100.0 & 100.0 \\
\cline { 2 - 7 } & SPE & 85.0 & 92.7 & 98.0 & 88.0 & 88.0 \\
\cline { 2 - 7 } & ACC & 79.8 & 93.1 & $\mathbf{9 8 . 1}$ & $\underline{88.4}$ & 88.4 \\
\hline \multirow{3}{*}{ Temporal } & SEN & 82.5 & 94.0 & 100.0 & 100.0 & 75.0 \\
\cline { 2 - 7 } & SPE & 87.0 & 93.8 & 100.0 & 95.7 & 87.9 \\
\cline { 2 - 7 } & ACC & 87.0 & 93.8 & $\mathbf{1 0 0 . 0}$ & $\underline{96.1}$ & 87.4 \\
\hline Average & ACC & 83.4 & 93.5 & $\mathbf{9 9 . 0}$ & $\underline{92.2}$ & 88.1 \\
\hline
\end{tabular}

The technique in [12] the second IMF provides better classification results when AM and FM bandwidth features are used for the small dataset [15]. We have conducted experiments using the first three IMFs using the [7] dataset as is shown in Table 1 where the first IMF gives the best result compared to other IMFs. For the RBF kernel, the proposed method outperforms the stateof-the-art method consistently and the classification accuracies using the proposed method are $98.1 \%$ for Frontal lobe and $100.0 \%$ for Temporal lobe respectively, whereas the classification accuracy using the state-of-artmethod [17] is $79.8 \%$ for Frontal lobe and $87.0 \%$ for Temporal lobe EEG signals for the dataset [7]. Thus, in terms of three different classification criteria such as sensitivity, specificity, and accuracy, the proposed method outperforms the state-of-the-art method with greater consistency. The classification consistency among three different criteria is very important for proper diagnosis. Note that the best performance and second best performance are highlighted in Table 1 using bold and underline respectively.

Parvez et al. [22] proposed a technique using a number of new features based on DCT and DWT transformations for classification of ictal and interictal EEG signals using dataset [7]. The experimental results showed that the maximum accuracy was $85.80 \%$ to classify ictal and interictal EEG signals [22]. Note that temporal correlation for feature extraction was not exploited in [22]. Different bands of frequencies can be produced by applying DWT [23]. Among the different bands, the Gamma frequency band $(30-60 \mathrm{~Hz})$ contains the most distinguishable characteristics among different types of the EEG signals [24]. The ability of DWT on classify preictal and interictal was verified, by using the Gamma frequency band signals. Table 1 shows these results under the heading Technique [22]. To compare the performance of the techniques we have applied the Technique [12] and [22] as well as the proposed technique in this paper using the preictal and interictal EEG signals in dataset [7]. Table 1 shows that the technique [22] with temporal correlation produces better classification results compared to the technique in [12]. It is also interesting to note that the proposed scheme outperforms both techniques [22] and [12]. In terms of average accuracy, the proposed scheme (the best accuracy among different IMFs), the existing-2 scheme [22] and the Bajaj et al. [12] scheme show $99.0 \%, 93.5 \%$, and $83.4 \%$ respectively for the large dataset [7].

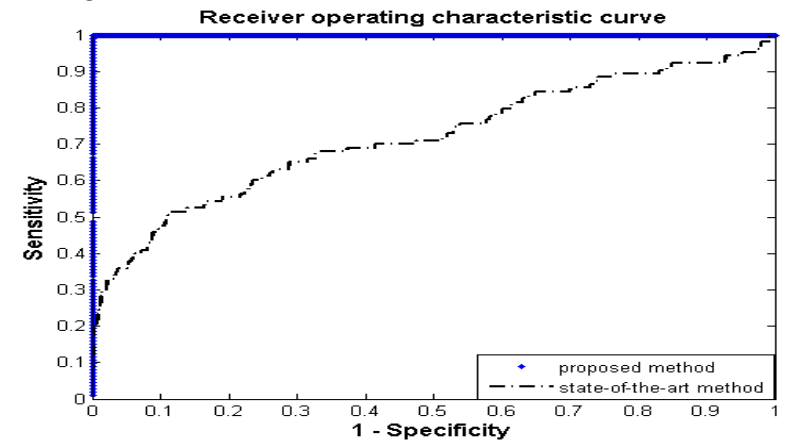

Fig. 5: The receiver operating characteristics (ROC) curves of the first IMF of training EEG signals by the proposed method against the stateof-the-art method using LS-SVM with RBF kernel from Temporal lobe.

The performance of the LS-SVM is evaluated by the receiver operating characteristics (ROC) plot shown in Fig. 5. ROC illustrates the performance of a binary classifier system where it is created by plotting the fraction of true positives from the positives i.e., true positive rate (TPR) vs. the fraction of false positives from negatives i.e., false positive rate (FPR) with various threshold settings. TPR is known as sensitivity, and FPR is one minus the specificity or the true negative rate. Fig. 5 demonstrates that the proposed method shows good classification results compared to the state-of-the-art method [12] using the dataset from the Temporal lobe of the first subset of the training EEG signals.

Fig. 6 represents classification comparisons using the proposed method and the state-of-the-art method [12]. Fig. 6 (b) and (c) show the LS-SVM classification results using the proposed EMD with DCT compared to the stateof-the-art method in Fig. 6(a). Fig. 6 shows that it is very 


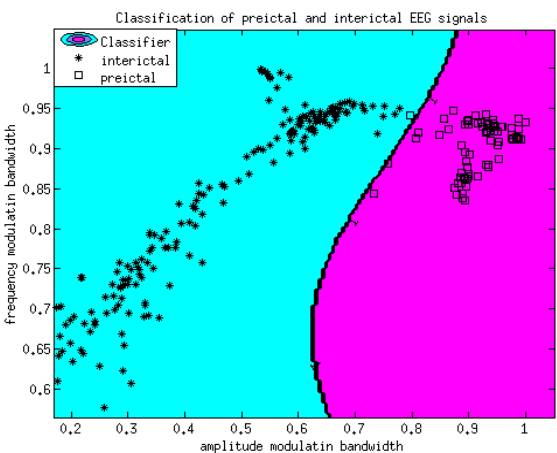

(a)

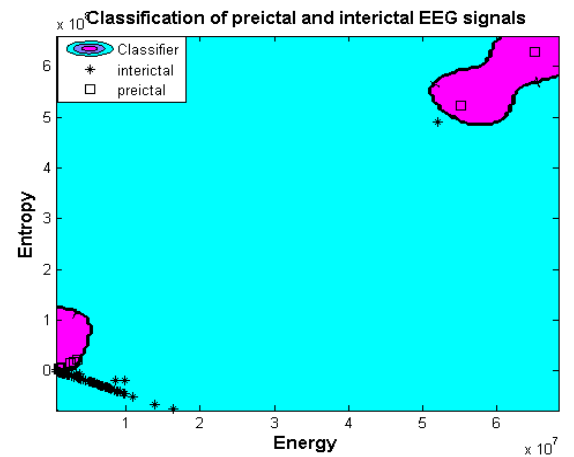

(b)

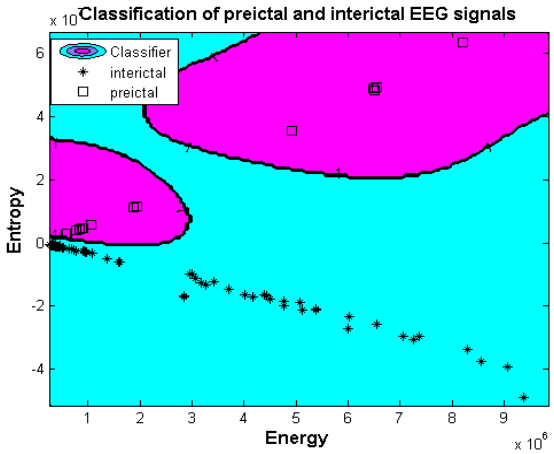

(c)

Fig. 6: Three images represent the classification of preictal and interictal EEG signals from Frontal lobe and Temporal lobe for the IMF1 of testing using (a) the state-of-the-art method for Frontal lobe (b) the proposed method for Frontal lobe and (c) the proposed method for Temporal lobe.

difficult to classify preictal and interictal EEG signals with having simple classifier and regular features. Therefore, we have extracted features using EMD and DCT and then LS-SVM classifier have used to classify them. It can be concluded from Table 1, Fig. 5 and Fig. 6 that the proposed method based on EMD with DCT outperforms the state-of-the-art method.

\section{CONCLUSION}

Temporal correlation provides seizure information and it carries the distinguishable features for preictal and interictal EEG signals classification. Therefore, in this paper we develop a method based on EMD and DCT by exploiting temporal correlation that used EEG signals to detect the pre-stage of epileptic seizure (i.e., preictal) using LS-SVM classifier. In the experiment, we get the $100 \%$ sensitivity (i.e., preictal) for Fontal and Temporal lobe EEG signals while state-of-the-art method provides $27.6 \%$ and $82.5 \%$ sensitivity for them. The experimental results also show that our proposed method perform more consistently in terms of sensitivity, specificity, and accuracy compared to the existing methods in different patients and different brain locations.

\section{REFERENCES}

[1] R. S. Fisher, W. van Emde Boas, W. Blume, C. Elger, P. Genton. P. Lee, and J. Jr. Engel," Epileptic seizures and epilepsy: definitions proposed by the International League Against Epilepsy and the International Bureau for Epilepsy (IBE)," Epilepsia, 46(4), 470-472, 2005

[2] P. Kwan, and M.J. Brodie," Refractory epilepsy: mechanisms an solutions," Expert Review of Neurotherapeutics, 6(3):397-406, 2006.

[3] J. Rasekhi, M. R. K. Mollaei, M. Bandarabadi, C. A. Teixeira, and A. Dourado," Preprocessing effects of 22 linear univariate features on the performance of seizure prediction methods," Journal of Neuroscience Methods, vol. 217, Issues 1-2, 9-16, 2013.

[4] J. R. Williamsona, D. W. Blissa, D. W. Brownea, and J. T. Narayananb," Seizure prediction using EEG spatiotemporal correlation structure," Journal of Epilepsy \& Behavior, vol. 25(2), 230-238, 2012.

[5] F. Duman, N. Ozdemir, and E. Yildirim," Patient Specific Seizure Prediction Algorithm Using HilbertHuang Transform," IEEE-EMBS International Conference on Biomedical and Health Informatics, Hong Kong and Shenzhen, China, 2012.

[6] L. Chisci, A. Mavino, G. Perferi, M. Sciandrone, C. Anile, G Colicchio, and F. Fuggetta," Real-Time Epileptic Seizure Prediction Using AR Models and Support Vector Machines," IEEE Transactions on Biomedical Engineering, vol. 57(5), 2010.

[7] EEG Data set from Epilepsy Center of the University Hospital of Freiburg,http://epilepsy.uni-freiburg.de/freiburg-seizure-predictionproject/eeg-database, Visited Date: June 10, 2012.
[8] R. Panda, P.S. Khobragade, P.D. Jambhule, S. N. Jengthe, P. R. Pal, and T. K. Gandhi, "Classification of EEG signals using wavelet transform and support vector machine for epileptic seizure diction," International Conference on Systems in Medicine and Biology, 405408, 2010.

[9] S. G. Dastidar, H. Adeli, and N. Dadmehr, "Mixed-band wavelet chaos-neural network methodology for epilepsy and epileptic seizure detection," IEEE Transactions on Biomedical Engineering, vol. 54(9), 1545-1551, 2007.

[10] H. Ocak, "Optimal classification of epileptic seizures in EEG using wavelet analysis and genetic algorithm," Signal Processing, vol. 88 (7), 1858-1867, 2008.

[11] K. Polat and S. Günes, "Classification of epileptiform EEG using a hybrid system based on decision tree classifier and fast Fourier transform," Applied Mathematics and Computation., vol. 187 (2), 1017-1026, 2007.

[12] V. Bajaj and R. B. Pachori, "Classification of Seizure and Non-seizure EEG signals using Empirical Mode Decomposition," IEEE Transaction on Information Technology in Biomedicine, 2012.

[13] V. Vapnik, "The nature of statistical learning theory," SpringerVerlag, New-York, 1995

[14] J. A. K. Suykens and J. Vandewalle," Least Squares Support Vector Machine Classifiers," Neural Processing Letters, vol. 9(3), 293-300, 1999.

[15] "Epilepsy data: a few small files (text format)," http://epileptologiebonn.de/cms/front_content.php?idcat=193\&lang=3\&changelang=3, Visited Date: April 28, 2012.

[16] TP. Jung, S. Makeig, C. Humphries, et al., "Removing electroencephalographics artifacts by blind source separation," Psychophysiology, 37, 163-78, 2000

[17] W. De Clercq, A. Vergult, B. Vanrumste, W. V. Paesschen, S. V. Huffel, "Canonical Correlation Analysis Applied to Remove Muscle Artifacts From the Electroencephalogram," IEEE Trans. Biomed. Engineering 53(12), 2583-2587, 2006.

[18] J. Ma, P. Tao, S. Bayram, V. Svetnik, "Muscle artifacts in multichannel EEG: characteristics and reduction," Clinical Neurophysiol, 123(8), 1676-86, 2012.

[19] S. Abe, Support vector machine for pattern classification, Second Edition, Springer, 2010.

[20] K. D. Brabanter, P. Karsmakers, F. Ojeda, C. Alzate, and J. D. Brabanter, K. Pelckmans, B. De Moor, J. Vandewalle, J.A.K. Suykens, LS-SVMlab Toolbox User's Guide, version 1.8, Katholieke Universiteit Leuven, August 2011.

[21] H. Wang and D. Hu," Comparison of SVM and LS-SVM for Regression," International Conference on Neural Networks and Brain, vol. 1, 279-283, 2005 .

[22] M. Z. Parvez and M. Paul, "Classification of Ictal and Interictal EEG signals," IASTED conference on Biomedical Engineering, pp. 791031,2013

[23] H. Adeli, Z. Zhou, and N. Dadmehr,"Analysis of EEG records in an epileptic patient using wavelet transform," Journal of Neuroscience Methods, vol. 123, 69-87, 2003.

[24] A. Keil, M. M. Muller,1 W. J. Ray, T. Gruber, and T. Elbert," Human Gamma Band Activity and Perception of a Gestalt," Journal of Neuroscience, 19(16),7152-7161, 1999. 